\title{
The effect of dimensional preference on optional shift behavior and concept attainment of retarded adolescents
}

\author{
RICHARD M. GARGIULO, TAD UNO, JAMES D. SEARS, and PAUL HISZEM \\ Bowling Green State University, Bowling Green, Ohio 43403
}

\begin{abstract}
Sixty-three moderately retarded adolescents of similar chronological and mental ages participated in an experiment designed to assess the influence of dimensional dominance and preference on two concept learning tasks. After dominance assessment, subjects were randomly assigned to either a preferred or nonpreferred condition and received both an optional shift and concept assessment test. The results indicated a significant difference between color- and form-dominant learners and between subjects assigned to preferred and nonpreferred dimensions. No difference was observed on the concept assessment measure. A significantly greater number of subjects solved the optional shift task intradimensionally. Intradimensional shifts were also learned faster than extradimensional shifts. The data were interpreted in terms of developmental and mediation hypotheses and viewed as consistent with Zeaman and House's chaining prediction.
\end{abstract}

Considerable attention has been devoted to the relationship between dimensional preference and performance on various conceptual tasks (Smiley, 1973; Suchman \& Trabasso, 1966b; Trabasso, Stave, \& Eichberg, 1969). Such a relationship is generally explained in terms of attention theory (Zeaman \& House, 1963). Zeaman and House hypothesized that a sequence of two responses is required to explain the performance of retarded children on visual discrimination tasks. Specifically, the learner must make an attentional (observing) response to the relevant stimulus dimension (e.g., color or form), as well as the correct instrumental approach response to the cues of that dimension (yellow or triangle). Zeaman and House further postulated that the probability of a subject observing the relevant stimulus dimension and approaching the correct stimulus cue is the result of reinforcement and is altered by various transfer paradigms such as intradimensional (ID) and extradimensional (ED) shifts.

Shift strategy is determined by the relationship established between the relevant stimulus cues of the original and transfer problems. The typical ID/ED shift experiment requires the changing of the relevant cues to a different pair of cues within the same dimension for an ID shift; an ED shift involves changing the

The authors gratefully acknowledge the helpful support of the students and staff of the Wood Lane School, Bowling Green, Ohio, and the Blanchard Valley Center, Findlay, Ohio, for their thoughtfulness and cooperation. The authors wish to thank Janet Sulick and Sue Benedict for their assistance in the conduct of this study. Appreciation is also extended to Ralph St. John for statistical consultation. Request for reprints should be sent to Richard M. Gargiulo, Department of Special Education, Bowling Green State University, Bowling Green, Ohio 43403. Tad Uno is now at Gallaudet College, MSSD, Kendall Green, Washington, D.C. 20002. James D. Sears is now at the Area of Special Education, University of Alabama, University, Alabama 35486 . relevant cues of a discrimination to those of a new dimension. For example, if red is chosen by the experimenter as the positive cue, changing the cue to yellow during transfer constitutes an ID shift, while switching to triangle exemplifies an ED shift.

Zeaman and House (1963) predicted that an ID shift would be learned faster than an ED shift. Experimental support for this fundamental prediction has been found with animals (Mackintosh \& Little, 1969), preschool children (Dickerson, 1966), grade-school children (Eimas, 1966), and retarded individuals (Campione, Hyman, \& Zeaman, 1965).

Considering the relationship between preference and concept attainment, Brown (1970) noted that dimensional preference is not only related to concept acquisition, but also plays a role in conceptual performance requiring a complex relational solution. Research evidence (Suchman \& Trabasso, 1966a) has suggested that children who prefer color are less efficient problem solvers than form-preferring youngsters, and colordominant children are "less mature, intelligent and advanced in general problem solving ability" (Brown, 1970, p. 249).

The present study investigated conceptual learning of moderately retarded individuals of similar mental ages. It was anticipated that dimensional preference would advantageously affect performance on both the optional shift and concept attainment tasks. Additionally, significant differences were not expected in the number of ID/ED shifts learned; however, it was predicted that ID shifts would be solved faster.

\section{METHOD}

Subjects
The subjects were 63 moderately retarded adolescents of
similar chronological (CA) and mental (MA) ages attending 
public day-school programs. Thirty-seven boys and 26 girls participated in the experiment. Five subjects failed to demonstrate dimensional dominance and two subjects were eliminated due to experimenter error. Therefore, 56 retarded individuals were involved in the experiment. The subjects did not demonstrate major sensory or motor deficiencies. Table 1 presents the mean MA, CA, and IQ of the subjects. IQ scores were based upon data obtained from a yearly psychological examination.

\section{Stimulus Materials}

Dimensional dominance was assessed using red and green circles and triangles presented on white $4 \times 6$ in. cards. Each drawing was approximately $1 \mathrm{sq}$ in.

The optional shift procedure required two sets of stimulus cards that varied in color and form: One set varied with color values of red and green and form values of circle and triangle; the second set varied with color values of blue and yellow and form values of square and cross. A total of 32 color-form patterns were generated. Each color and form value was presented on a white $4 \times 6$ in. card. Spatial arrangements of the stimuli were randomly determined.

Concept attainment was directly assessed by administering the first three tests of the "Concept Development Assessment Tests: Equilateral Triangle" (Klausmeier, Ingison, Sipple, \& Katzenmeyer, Note 1). This instrument is based on a model of conceptual learning and development proposed by Klausmeier, Ghatala, and Frayer (1974). A total of 19 test items were individually administered on white $4 \times 6$ in. cards. The first test required only the discrimination of an equilateral triangle from other geometric figures (e.g., squares, rectangles, and other triangles). The distractors varied in shape, color, size, and number. The second test involved discriminating various forms of an equilateral triangle from the distractors, as well as generalizing the forms as equivalent. The final test required the subject to generalize that different instances of an equilateral triangle were equivalent in some manner.

\section{Procedure}

Individual subjects were escorted to a quiet, windowless experimental room and were told that they were to play a "guessing game." Each subject sat directly opposite a female experimenter. All stimulus materials were concealed behind a small screen.

\section{Dominance Assessment}

Testing began 1 week prior to the presentation of either the optional shift problem or the concept task. The testing procedure closely followed the method of triads reported by Trabasso et al. (1969). The 18 cards were presented to the subject one at a time, beginning with the redundant cue card. The subject was asked to "point to the two pictures that look alike," with the experimenter demonstrating. Extensive verbal reinforcement was given when the subject indicated a correct choice. If an incorrect response was made the experimenter said, "No, these two are alike," and pointed to the two identical figures. The five remain-

Table 1

Mean Subject MA, CA, and IQ

\begin{tabular}{|c|c|c|c|c|c|c|c|}
\hline & \multirow[b]{2}{*}{$\mathrm{N}$} & \multicolumn{2}{|c|}{ MA } & \multicolumn{2}{|c|}{ CA } & \multicolumn{2}{|c|}{ IQ } \\
\hline & & Mean & SD & Mean & SD & Mean & SD \\
\hline & \multicolumn{7}{|c|}{ Color } \\
\hline $\begin{array}{l}\text { Preferred } \\
\text { Nonpreferred }\end{array}$ & $\begin{array}{l}12 \\
12\end{array}$ & $\begin{array}{l}4.44 \\
4.29\end{array}$ & $\begin{array}{r}1.17 \\
.78\end{array}$ & $\begin{array}{l}11.82 \\
12.66\end{array}$ & $\begin{array}{l}4.38 \\
1.42\end{array}$ & $\begin{array}{l}42.87 \\
45.87\end{array}$ & $\begin{array}{l}5.35 \\
4.76\end{array}$ \\
\hline & \multicolumn{7}{|c|}{ Form } \\
\hline $\begin{array}{l}\text { Preferred } \\
\text { Nonpreferred }\end{array}$ & $\begin{array}{l}12 \\
12\end{array}$ & $\begin{array}{l}4.33 \\
4.20\end{array}$ & $\begin{array}{l}1.97 \\
1.24\end{array}$ & $\begin{array}{l}12.68 \\
12.31\end{array}$ & $\begin{array}{l}2.82 \\
2.89\end{array}$ & $\begin{array}{l}38.75 \\
46.50\end{array}$ & $\begin{array}{l}5.30 \\
6.19\end{array}$ \\
\hline
\end{tabular}

Note-The intelligence quotients were derived from either the Stanford-Binet or Weschler Intelligence Scale for Children. ing redundant cue cards were successively presented with the subject self-pacing his choices. Upon completion of the redundant cards, 12 cards were successively exhibited which presented opposed color and form values. Verbal reinforcement was not provided during this series. Subjects responding 10 out of 12 times to color were judged to be color dominant; subjects who chose form to the same ratio were considered to be form dominant. Subjects not obtaining this ratio were considered inconsistent and were eliminated from further consideration. Upon completion of the assessment, subjects were escorted back to their classroom.

Prior to the presentation of the experimental task, the subjects were assigned to either a color- or a form-dominant group, based upon the assessment results. Twenty-four subjects were randomly chosen from a group of 29 color-dominant individuals and 24 form-dominant adolescents were randomly selected from a group of 27 . One-half of the subjects in each of the two groups were then randomly assigned to either a preferred or a nonpreferred condition. The resulting design was a 2 by 2 factorial with dimension (color or form) as the first variable and preference (preferred or nonpreferred) as the second variable. One-half of the subjects received the optional shift task first; the remaining subjects received the concept assessment test first.

\section{Original Learning}

The initial training and optional shift problem followed the general procedure outlined by Kendler, Kendler, and Ward (1972). The two-choice visual discrimination task began with the experimenter manually presenting a stimulus card. The subject was instructed to point to the correct stimulus. The subject's responses were verbally reinforced by the experimenter. The remaining stimulus cards were presented at 5-sec intervals. The irrelevant dimension varied within trials, each cue was positive and negative an equal number of times. A noncorrection procedure was employed throughout the experiment.

All subjects were trained to a criterion of 10 consecutive correct responses or 50 trials. If the subject failed to learn within 50 trials, the session was terminated and begun again the next day the subject was available. Special training procedures were not employed.

\section{Optional Shift}

Immediately after the subject reached criterion on the original discrimination, the optional shift problem was presented. For each subject, entirely new color and form stimuli were introduced. The subject was now required to learn a shift discrimination in which color and form were redundant and relevant. The subject was trained to the same criterion employed during the initial learning task.

Upon obtaining criterion, a 20 -trial test series was immediately introduced to discover on what basis the shift discrimination was solved. On a random 10 of these trials, the relearning trials, the pair of stimuli employed during the shift problem was presented and the reward contingencies remained the same. On the remaining 10 test trials, the color and form cues were re-paired, with each spatial setting appearing five times and all responses reinforced. If on these trials the subject chose the positive cue from the dimension that was relevant on the initial discrimination eight or more times, he was said to have made an ID choice; if the subject selected the positive cue from the dimension that was initially irrelevant eight or more times, the shift was classified as an ED choice. Those subjects who failed to make eight or more consistent responses were classified as inconsistent (I). Upon completion of the optional shift problem, subjects received a candy bar and were returned to their classroom.

\section{Concept Assessment}

Subjects were administered the three concept development tests (Klausmeier et al., Note 1) according to procedures outlined in the administrators' manual. One procedural modification was made: Subjects were instructed to point to the correct 
response rather than marking it with a felt-tipped pen. Responses were scored as correct or incorrect.

\section{RESULTS}

To correct for heterogeneity of variance, all analyses were conducted using natural logarithmic transformations of the raw data. The dependent variable was log trials to criterion +1 .

A multivariate analysis of covariance (Finn, Note 2) was used to assess the overall effect of dimensional dominance and preference on the original learning and optional shift tasks. The covariance procedure was used to eliminate the effect of mental age.

The multivariate procedure indicated a significant main effect for dimensional dominance $[F(2,42)=$ $70.6964, \mathrm{p}<.0001]$. This analysis also suggested that subjects assigned to their preferred dimensions, differed significantly in learning from subjects assigned to the nonpreferred conditions $[\mathrm{F}(2,42)=47.1747, \mathrm{p}<$ $.0001]$. The Dimension by Preference interaction was nonsignificant.

Since the multivariate analysis indicated significance, univariate analysis of covariance tests were conducted, as recommended by McCall (1970). The univariate $F$ tests indicated significance for the variables of color vs. form and preferred vs. nonpreferred on the initial discrimination and shift tasks $(\mathrm{p}<.0001)$.

Table 2 illustrates the mean values associated with each dimension and preference combination. Examination of the table indicated that form-dominant retarded adolescents learned faster than their color-dominant counterparts. Also, subjects assigned to the preferred dimension performed significantly faster than individuals assigned to the nonpreferred dimension.

A one-way ANOVA suggested no difference between color-dominant (mean $=2.23$ trials) and form-dominant (mean $=2.39$ trials) learners on the concept measure $[\mathrm{F}(1,46)=2.47, \mathrm{p}>.12]$.

Performance on the original discrimination and optional shift task was also examined in terms of how the tasks were solved (i.e., ID or ED) and the number of trials to criterion. Assuming an equal chance of solving the shift task either intradimensionally or extradimensionally (i.e., probability $=.50$ ), a binomial distribution indicated that a significantly greater $(p<.03)$ number of learners solved the shift discrimination intradimensionally (ID $=29$ vs. $E D=16)$. The three inconsistent subjects (two color nonpreferred and one form nonpreferred) were eliminated from analysis. Nonpreferred subjects differed significantly in the number of ID and ED shifts solved (16 and 5, respectively; $\mathrm{p}<.01)$; in particular, form-nonpreferred subjects solved the task with a greater number of ID shifts ( 9 vs. $2 ; p<.03)$. No other dominance by preference combinations revealed significant differences in the number of ID/ED shifts solved.

A three-way ANOVA supported the findings of the multivariate analysis and suggested that on the original learning task, ID subjects (mean $=3.26$ trials) learned significantly faster than ED subjects (mean $=3.39$ trials) $[F(1,37)=34.31, p<.001]$. No significant interactions were indicated. When the ID and ED shift averages were adjusted for the effect of dominance, preference, and unequal cell size (Nie, Hull, Jenkins, \& Steinbrenner, 1975), ID shifts (mean $=2.53$ trials) were also solved faster than ED shifts (mean $=2.61$ trials) on the optional shift task $[F(1,37)=4.62$, $\mathrm{p}<.03]$. Significant Dominance by Preference and Dominance by Shift interactions were evident, however,

The effect of dominance influenced performance according to whether subjects were assigned to a preferred or nonpreferred condition. Although form subjects learned the shift task faster, the difference between the color-preferred (mean $=2.60$ trials) and colornonpreferred subjects (mean $=2.94$ trials) was approximately twice as large as the difference between the form-preferred (mean $=2.27$ trials) and formnonpreferred subjects (mean $=2.45$ trials) $[F(1,37)=$ $6.90, \mathrm{p}<.01]$. The Dominance by Shift interaction suggested that subjects assigned to color solved the ID shift (mean $=2.71$ trials) faster than the ED shift (mean $=2.82$ trials), but form subjects learned the ED shift (mean $=2.31$ trials) faster than the ID shift $($ mean $=2.39$ trials) $[\mathrm{F}(1,37)=5.66, \mathrm{p}<.02]$.

\section{DISCUSSION}

The present findings supported the hypothesis that colorpreferring children are less efficient problem solvers (Suchman \& Trabasso, 1966a; Trabasso et al., 1969), possibly indicating that preference for a dimension is a correlate of cognitive growth, that is, that form-dominant learners function at a higher conceptual level. However, contrary to Brown's (1970) research, no difference was evident between color- and form-dominant adolescents on the concept attainment measure. This result might be due to the nature of the task. One could speculate that in comparison to the shift discrimination, the concept attainment test provided a more accurate indicator of the cognitive abilities of moderately retarded individuals in that

Table 2

Mean Scores According to Dimensional Dominance and Preference

\begin{tabular}{|c|c|c|c|c|c|c|c|c|c|c|c|c|c|c|c|c|c|c|}
\hline \multirow[b]{3}{*}{. } & \multicolumn{9}{|c|}{ Original Learning } & \multicolumn{9}{|c|}{ Optional Shift } \\
\hline & \multicolumn{3}{|c|}{ Color } & \multicolumn{3}{|c|}{ Form } & \multicolumn{3}{|c|}{ Mean Total } & \multicolumn{3}{|c|}{ Color } & \multicolumn{3}{|c|}{ Form } & \multicolumn{3}{|c|}{ Mean Total } \\
\hline & $\mathbf{N}$ & Mean & SD & $\mathrm{N}$ & Mean & SD & $\mathrm{N}$ & Mean & SD & $\mathbf{N}$ & Mean & SD & $\mathbf{N}$ & Mean & SD & $\mathbf{N}$ & Mean & SD \\
\hline Preferred & 12 & 3.33 & .20 & 12 & 3.05 & .10 & 24 & 3.19 & .21 & 12 & 2.60 & .12 & 12 & 2.27 & .12 & 24 & 2.44 & .20 \\
\hline Nonpreferred & 12 & 3.58 & .13 & 12 & 3.33 & .09 & 24 & 3.45 & .16 & 12 & 2.94 & .09 & 12 & 2.48 & .15 & 24 & 2.71 & .26 \\
\hline Mean Total & 24 & 3.45 & .21 & 24 & 3.19 & .17 & 48 & 3.32 & .23 & 24 & 2.77 & .20 & 24 & 2.38 & .16 & 48 & 2.57 & .27 \\
\hline
\end{tabular}

Note-Original learning and optional shift scores are expressed in trials to criterion. All scores are based upon transformed data. 
more complex relational solutions were required.

It could also be argued that a developmental component influenced performance. That is, a transition occurs at approximately age 4 years and reaches its maximum at 4.5 years, with children undergoing a change from color to form preference (Brian \& Goodenough, 1929; Suchman \& Trabasso, 1966a). As the mental ages of the subjects in the present experiment approximated this chronological age range, it is plausible that the children were in a transitional stage and hence uncertain about their "true preference," which may have contributed to the present findings.

Learning was more rapid on the shift discriminations when subjects were assigned to their preferred dimensions (Trabasso et al., 1969). It may be that a learner prefers a dimension because he can abstract it better than he can abstract the nonpreferred dimension. Whether the relevant dimension (color or form) is congruent with the learner's preferred dimension has a greater effect on the shift behavior of younger (mentally immature) subjects (Glick, Note 3). The performance of the preferred subjects may also be interpreted as evidence for a selective attention factor that suggests the inability of the subjects assigned to the nonpreferred conditions to inhibit attention to the preferred but irrelevant cue.

Contrary to expectations, a significantly greater number of subjects solved the shift task intradimensionally. This result was unexpected due to the restricted mental ability level investigated, which would appear to argue against an ontogeny of shift development. However, the finding that $64 \%$ of the subjects chose an ID shift is consistent with Campione's (1970) data and in agreement with the Kendler et al. (1972) argument that individuals prefer an ID shift in an optional shift paradigm. The present experiment can also be interpreted as supporting the Kendler et al. mediation hypothesis that an ID choice is indicative of a mediational response. Once could further assume that subjects who responded extradimensionally were ineffective in their use of verbal mediators, possibly due to a deficiency in verbal mediation (Reese, 1962). A complementary explanation of a production deficiency (Flavell, Beach, \& Chinsky, 1966) hypothesizes that the subjects' habitual failure to produce the mediators at the proper time effected performance. However, whether the performance of the ED subjects was due to a mediational or production deficiency remains obscure.

Similarly, if the subject learned a mediating response to the relevant (preferred) dimension of the original discrimination, then this response should transfer to the shift task and the ID shift should be easier than an ED shift (Campione, 1970). One would further expect an ED shift to be easier if the subject's preferred dimension is irrelevant. However, this assumption was not supported in the present study. Apparently, some of the nonpreferred subjects learned a mediating response to the relevant dimension, which resulted in a preference for an ID shift. This may possibly be attributed to the subjects' uncertainty regarding their dimensional preferences.

The results were consistent with the Campione et al. (1965) shift research with moderately retarded children, and they were viewed as supporting the Zeaman and House (1963) chaining prediction that an ID shift would be learned faster than an ED shift. One could speculate that the original learning problem resulted in an increased probability of attending to the relevant dimension and that this attending process transferred to the optional shift task, which resulted in the superior performance of the ID subjects (Campione, 1973). If the shift discrimination had been learned in a manner consistent with a single-unit S-R theory (Spence, 1936), no difference would have existed between the ID and ED shifts.

The Dominance by Preference interaction evidenced on the optional shift discrimination would appear to support an earlier assumption that form-dominant individuals are more efficient problem solvers. However, the Dominance by Shift interaction suggested that form subjects experienced difficulty in learning an appropriate mediating response to the shift task. Also, the possible unreliability of the subject's dimensional preference must be considered.

\section{REFERENCE NOTES}

1. Klausmeier, H., Ingison, L., Sipple, T., \& Katzenmeyer, C. Development of conceptual learning and development assessment series I: Equilateral triangle (Working Paper 119). Unpublished manuscript, Wisconsin Research and Development Center for Cognitive Learning, 1973.

2. Finn, J. Multivariance-univariate and multivariate analysis of variance and covariance: A FORTRAN IV program (Version 5). Unpublished manuscript, State University of New York at Buffalo, 1972.

3. Glick, J. Mediation and transfer in concept formation. (Final Progress Report, Grant MY 11886). University of Minnesota, Institute of Child Development, 1966.

\section{REFERENCES}

Brian, C., \& Goodenough, F. Relative potency of color and form perception at various ages. Journal of Experimental Psychology, 1929, 12, 197-213.

Brown, A. The stability of dimensional preference following oddity training. Journal of Experimental Child Psychology, 1970, 9, 239-252.

Campione, J. Optional intradimensional and extradimensional shifts in children as a function of age. Journal of Experimental Psychology, 1970, 84, 296-300.

Campione, J. The generality of transfer: Effects of age and similarity of training and transfer tasks. Journal of Experimental Child Psychology, 1973, 15, 407-418.

Campione, J., Hyman, L., \& Zeaman, D. Dimensional shifts and reversals in retardate discrimination learning. Journal of Experimental Child Psychology, 1965, 2, 255-263.

Dickerson, D. Performance of preschool children on three discrimination shifts. Psychonomic Science, 1966, 4, 417-418.

Eimas, P. Effects of overtraining and age on intradimensional and extradimensional shifts. Journal of Experimental Child Psychology, 1966, 3, 348-355.

Flavell, J., Beach, D., \& Chinsky, J. Spontaneous verbal rehearsal in a memory task as a function of age. Child Development, 1966, 37, 283-299.

Kendler, H., Kendler, T., \& Ward, J. An ontogenic analysis of optional intradimensional and extradimensional shifts. Journal of Experimental Psychology, 1972, 95, 102-109.

Klausmeier, H., Ghatala, E., \& Frayer, D. Conceptual learning and development. New York: Academic Press, 1974.

Mackintosh, N., \& LitTle, L. Intra- and extradimensional shift learning by pigeons. Psychonomic Science, 1969, 14, 5-6.

McCALL, $R$. The use of multivariate procedures in developmental psychology. In P. Mussen (Ed.), Carmichael's manual of child psychology (Vol. 1). New York: Wiley, 1970.

Nie, N. H., Hull, C. H., Jenkins, J. G., Steinbrenner, K., \& BENT, D. H. Statistical package for the social sciences. New York: McGraw-Hill, 1975.

ReEse, H. Verbal mediation as a function of age. Psychological Bulletin, 1962, 59, 502-509.

Smiley, S. Optional shift behavior as a function of age and dimensional preference. Journal of Experimental Child Psychology, 1973, 16, 451-458.

SPENCE, K. The nature of discrimination learning in animals. Psychological Review, 1936, 43, 427-449.

Suchman, R., \& Trabasso, T. Color and form preference in young children. Journal of Experimental Child Psychology, 1966, 3, 177-187. (a)

Suchman, R., \& Trabasso, T. Stimulus preference and cue function in young children's concept attainment. Journal of Experimental Child Psychology, 1966, 3, 188-198. (b)

Trabasso, T., Stave, M., \& Eichberg, R. Attribute preference and discrimimination shifts in young children. Journal of $E x$ perimental Child Psychology, 1969, 8, 195-209.

Zeaman, D., \& House, B. An attention theory of retardate discrimination learning. In N. Ellis (Ed.), Handbook of mental deficiency. New York: McGraw-Hill, 1963.

(Received for publication May 23, 1979.) 